\title{
DESENHO UNIVERSAL PARA APRENDIZAGEM E TECNOLOGIA ASSISTIVA: COMPLEMENTARES OU EXCLUDENTES?
}

\author{
DISEÑO UNIVERSAL PARA EL APRENDIZAJE Y TECNOLOGÍA DE ASISTENCIA: \\ ¿COMPLEMENTARES O EXCLUYENTES?
}

\section{UNIVERSAL DESIGN FOR LEARNING AND ASSISTIVE TECHNOLOGY: COMPLEMENTARY OR EXCLUDING?}

\author{
Amália Rebouças de Paiva e OLIVEIRA ${ }^{1}$ \\ Adriana Garcia GONÇALVES ${ }^{2}$ \\ Lígia Maria Presumido BRACCIALI ${ }^{3}$
}

RESUMO: Com os avanços de pesquisas e legislações sobre a inclusão educacional surgiram inúmeras teorias e ações com o objetivo de favorecer a inclusão dos alunos com deficiência no contexto escolar, entre elas destacamos o Desenho Universal para Aprendizagem (DUA) e o uso da Tecnologia Assistiva (TA). Esse artigo apresenta uma discussão teórica pautada nos princípios que compõem o DUA e a TA, para tanto dissertamos como a literatura tem apontado que essas perspectivas, apesar de a priori parecerem incompatíveis, podem favorecer e ser complementares no processo de inclusão educacional.

PALAVRAS-CHAVE: Desenho universal para aprendizagem. Tecnologia assistiva. Inclusão escolar.

RESUMEN: Con los avanzos de las encuestas y legislaciones sobre la inclusión educacional surgieron muchas teorías y acciones con el objetivo de favorecer la inclusión de los alumnos con discapacidad en el contexto escolar, entre ellas destacamos el Diseño Universal para el Aprendizaje (DUA) y el uso de la Tecnología de Asistencia (TA). Ese artigo presenta una discusión teórica con base en los principios que componen el DUA y la TA, para tanto disertamos como la literatura hay apuntado que esas perspectivas, a pesar de a priori parecer incompatibles, pueden favorecer y ser complementares en el proceso de inclusión educacional.

PALABRAS CLAVE: Diseño universal para el aprendizaje. Tecnología de asistencia. Inclusión escolar.

${ }^{1}$ Universidade Federal de São Carlos (UFSCAR), São Carlos - SP - Brasil. Doutoranda no Programa de PósGraduação em Educação Especial (PPGEES). ORCID: https://orcid.org/0000-0002-8190-812X. E-mail: amaliareboucas@gmail.com.

${ }^{2}$ Universidade Federal de São Carlos (UFSCAR), São Carlos - SP - Brasil. Docente do Departamento de Psicologia (DPsi) e do Programa de Pós-Graduação em Educação Especial (PPGEES). ORCID: https://orcid.org/0000-0002-5725-2001. E-mail: adrigarcia@ufscar.br

3 Universidade Estadual Paulista (UNESP), Marília - SP - Brasil. Doutora em Educação Física. ORCID: https://orcid.org/0000-0002-2540-3725. E-mail: ligia.braccialli@unesp.br

RIAEE - Revista Ibero-Americana de Estudos em Educação, Araraquara, v. 16, n. esp. 4, p. 3034-3048, dez. 2021. e-ISSN: 1982-5587 
ABSTRACT: With the advances in researches and legislation about educational inclusion a lot of theories and actions have emerged in order to support the inclusion of students with disabillitis in the school context, among them we highlight the Universall Design for Learning (UDL) and the use of Assistive Technology (AT). This article presents a theoretical discussion based on the principles of Universal Design for Learning and Assistive Technology, to this end we discuss how the literature has pointed out that these perspectives, although they seem incompatible at first sight, they can favor and be complementary in the process of educational inclusion.

KEYWORDS: Universal design for learning. Assistive tecnology. School inclusion.

\section{Introdução}

A partir dos anos 90, século XX, o movimento de inclusão social e educacional ganha consistência no cenário brasileiro. Impulsionado, principalmente, por documentos internacionais como a Declaração de Salamanca (1994), que o Brasil foi signatário, e documentos nacionais como a Constituição da República Federativa do Brasil (BRASIL, 1988) e a Lei de Diretrizes e Bases da Educação Nacional (BRASIL, 1996).

O movimento pró-inclusão advindo de estudiosos, congressos, convenções, e pela própria legislação, principalmente, após a promulgação da Política Nacional da Educação na perspectiva da Educação Inclusiva (BRASIL, 2008), impacta diretamente no aumento ${ }^{4}$ do número de alunos público-alvo da Educação Especial (PAEE) na rede regular de ensino (VALENTINE; GOMES; BISOL, 2016).

Embora esse aumento de matrículas seja significativo e tenha contribuído para que os alunos PAEE ganhassem visibilidade no contexto escolar, é necessário pensar que apenas o acesso à escola regular não promove a inclusão desses alunos e não garante que eles tenham uma aprendizagem efetiva, de fato. Dados recentes da Unesco (2018) alertam que ao tratar da inclusão escolar a grande maioria dos países aborda apenas o acesso desses alunos à escola regular.

Nesse sentido, emerge a necessidade de teorias e práticas que subsidiem o professor a promover a inclusão no contexto educacional, pensando não apenas no acesso, mas na permanência e na aprendizagem do aluno PAEE na escola. Pesquisadores e teóricos passam a estudar, então, por meio de práticas baseadas em evidências, como garantir ao alunado PAEE seu direito à educação. Entre várias teorias e práticas estão os estudos sobre a Tecnologia Assistiva (TA) e o Desenho Universal para Aprendizagem (DUA).

${ }^{4}$ Censo Escolar Inep/MEC, 2019. Número de matrículas em classes comuns de alunos PAEE no ano de 2008: 376 mil; em 2019: 1 milhão e 91 mil. 
Em suma, essas duas vertentes se preocupam em promover o ensino, a acessibilidade e a inclusão de pessoas com deficiência no campo educacional e/ou social. Entretanto, trata-se de duas propostas que se desenvolveram paralelamente, e devido a isso, alguns estudiosos começaram a questionar se a área da TA contradiz os princípios estabelecidos pelo DUA.

Este artigo visa analisar as características do DUA e da TA por meio de uma discussão teórica, e compilar suas similaridades e divergências para o contexto educacional. Para tanto, esse manuscrito subdivide-se em quatro partes, a saber: considerações sobre o DUA; considerações sobre a TA; DUA e TA: aproximações e distanciamentos conceituais e práticos; e Considerações finais.

\section{Considerações sobre o DUA}

O conceito de Desenho Universal para Aprendizagem (Universal Design for Learning) surgiu em 1999 nos Estados Unidos, proposto por David Rose e Anne Meyer, pesquisadores que faziam parte do "Center for apllied special technology" (NELSON, 2014). Em suma, o conceito do DUA consiste em elaborar estratégias de acessibilidade facilitada a todos, no que se refere a estruturas físicas, serviços, produtos e soluções educacionais. Dessa maneira, pensa-se em múltiplos meios de oferecer a aprendizagem, reduzindo assim as barreiras impostas pelo ambiente educacional tradicional (CAST, 2013).

Nelson (2014) ressalta que o conceito do DUA não foi pensado, especificamente, para as pessoas com deficiência, mas sim em uma maneira de oferecer o ensino a todos os estudantes. A autora ainda aponta que este conceito tem como base a neurociência, partindo do pressuposto de que cada indivíduo aprende de maneira diferente, ou seja, o que pode ser uma experiência significativa para um, pode não ser eficaz para proporcionar a aprendizagem de outro. Alves, Ribeiro, Simões (2013) corroboram ao afirmar que o DUA é uma alternativa para pensar em diferentes maneiras de ensinar o mesmo currículo a todos os estudantes.

A teoria do DUA vem então ao encontro das ciências das aprendizagens, pensando em planejar e aplicar estratégias diversas que atinjam todos os alunos, para que cada um com sua especificidade processe a aprendizagem da maneira mais eficaz. Afinal, "quanto maior as possibilidades de apresentar um novo conhecimento, maior as possibilidades de aprendê-lo" (ZERBATO, 2018, p. 58).

Rose e Meyer (2014) abordam que o DUA é composto por três grandes princípios; são eles: princípio do engajamento; princípio da representação; princípio da ação e expressão. 
Estes princípios são norteadores para desenvolver um planejamento e estratégias que levem a uma atividade inclusiva.

O princípio do engajamento deve orientar o professor a pensar em como engajar os alunos na atividade, ou seja: Como desafiar esses alunos e mantê-los interessados? Quais estratégias o professor pode utilizar para motivar os alunos a participar da aprendizagem? Está relacionado às redes afetivas dos alunos, em assimilar o porquê da aprendizagem (NELSON, 2014).

No segundo princípio, deve-se pensar em como apresentar a informação aos estudantes (ROSE; MEYER, 2014). Trata-se do princípio da representação, no qual o aluno deverá reconhecer o "o quê" da aprendizagem. É importante no planejamento pensar em como apresentar as mesmas informações e conteúdos de diferentes maneiras (NELSON, 2014).

Nelson (2014) exemplifica ainda que o terceiro e último princípio, intitulado de Ação e Expressão, orienta os professores a auxiliar o aluno a processar e organizar aquilo que foi aprendido. Dessa maneira, o aluno poderá expressar de seu jeito o conhecimento adquirido em determinada tarefa. Refere-se ao "como" da aprendizagem. A autora destaca ainda que a flexibilidade é fundamental para que o aluno consiga expressar e ressignificar sua aprendizagem.

Diante de todo o exposto, percebe-se que o DUA é uma abordagem teórica que visa oferecer subsídios aos professores para pensar em atividades para todos. Não se trata de atividades específicas ou adaptações de ensino, mas sim em fornecer caminhos diferentes para que o professor possa pensar na aprendizagem do aluno. É uma maneira de romper com o tradicional currículo engessado, padronizado e denominado por Rose e Meyer (2014) de currículo tamanho único.

A sólida teoria constituída pelo DUA promete auxiliar na inclusão, tornando o ensino mais acessível a todos (NELSON, 2014; NUNES; MADUREIRA, 2015; RIBEIRO; AMATO, 2018; ROSE; MEYER, 2014). Em consonância, pesquisas práticas têm demonstrado a efetividade dos princípios do DUA no planejamento de aulas e atividades inclusivas em diversas disciplinas (ZERBATO, 2018). Entretanto, é necessário ressaltar que embora seja um conceito criado em 1999 e bastante estudado na América do Norte (OLIVEIRA; MUNSTER; GONÇALVES, 2019), no Brasil as pesquisas com essa temática ganharam força nos últimos 10 anos (PRAIS; ROSA, 2017). 


\section{Considerações sobre a Tecnologia Assistiva}

A Tecnologia Assistiva é caracterizada como uma área de conhecimento multidisciplinar, que por meio de recursos, metodologias, estratégias, práticas e serviços visa promover independência, autonomia, qualidade de vida e inclusão social para pessoas com deficiências, e/ou mobilidade reduzida (BRASIL, 2015). Manzini e Deliberato (2007) elencam três elementos que auxiliam a compreender o conceito de TA: 1) é uma tecnologia a serviço de pessoas com deficiência; 2) ela deve promover funcionalidade; 3) deve servir para a inclusão social.

Embora a TA pertença a um campo multidisciplinar extremamente amplo, este texto irá focar na TA no cenário educacional. Isso porque o objetivo deste artigo concentra-se em um olhar específico para o DUA e a TA como facilitadores para a inclusão educacional.

Toyoda e Lourenço (2008) apontam que o interesse pela área da TA no Brasil surgiu em meados da década de 70, entretanto sua ligação com o contexto educacional começou a ser estudada na década de 90 (CALHEIROS; MENDES; LOURENÇO, 2018). A TA encontra-se no universo escolar de duas formas: a primeira são os recursos de TA que compõem as salas multifuncionais (o que se denomina de recurso de TA de alto custo), e os recursos de TA de baixo custo, aqui compreendidos como "produtos pré-fabricados e de baixo custo e adaptações confeccionados com material alternativo" (HOHMANN; CASSAPIAN (2011, p. 10), que na maioria das vezes se apresentam em forma de adaptações realizadas pelos professores, terapeutas ocupacionais e fisioterapeutas.

Deste modo, Calheiros, Mendes e Lourenço (2018, p. 231) salientam que:

No espaço educacional, com destaque para as práticas realizadas diretamente com a população-alvo da Educação Especial fortemente ainda nos contextos institucionais, a prática de diferenciação de materiais e uso de adaptações para a realização de atividades também era presente, por exemplo, na educação de pessoas com deficiência visual.

Essas adaptações realizadas na escola acontecem, na maioria das vezes, na atividade, e/ou no recurso, e/ou nas estratégias utilizadas pelo professor. Faz-se necessário resgatar, então a concepção de estratégias de ensino e recursos pedagógicos. O termo estratégia de ensino é concebido como a ação propriamente dita do professor e o recurso pedagógico é caracterizado por ser concreto, manipulável e possuir uma finalidade pedagógica (MANZINI; DELIBERATO, 2007). Ambos os conceitos fazem parte do campo multidisciplinar da TA e têm sido amplamente investigados em pesquisas, por funcionarem como elementos que facilitam o acesso do aluno PAEE a uma atividade no ambiente escolar (FIORINI, 2011). 
Ou seja, a estratégia de ensino utilizada pelo professor para passar determinado conteúdo pode sofrer alterações, de acordo com as necessidades do aluno, assim como o recurso pedagógico. Afinal, toda adaptação deve ser realizada sempre pensando em promover maior funcionalidade ao aluno, seja a adaptação no recurso propriamente dito, e /ou no ensino (SEABRA JUNIOR, 2008). Nesse sentido, diversos estudos têm apontado para os benefícios do uso da TA dentro da escola, seja ela para promover acessibilidade àqueles que possuem alguma limitação motora (ALVES; MATSUKURA, 2011), para colaborar com a comunicação alternativa no contexto escolar (MASSARO; DELIBERATO, 2013), e também na utilização de estratégias de ensino e recursos pedagógicos que auxiliem o aprendizado e/ou a inclusão desses alunos (FIORINI, 2011).

É de extrema importância que a implementação de um recurso na escola seja acompanhada pelo próprio estudante, afinal, é ele quem irá usufruir dos benefícios dessa tecnologia, e que poderá dizer se o recurso é funcional ou não às suas necessidades (ALVES; MATSUKURA, 2011; LOURENÇO, 2012). Além do que, "o entendimento do contexto e situação do aluno com deficiência na escola é fundamental para a prescrição, construção, adaptação e implementação dos recursos de tecnologia assistiva" (ROCHA; DELIBERATO; LAMÔNICA, 2012, p. 87).

Por fim, alguns estudos têm apontado empecilhos que dificultam a utilização da TA no contexto escolar. Calheiros, Mendes e Lourenço (2018) apontam que uma grande barreira diz respeito à formação de recursos humanos para trabalhar com essa tecnologia, ou seja, os professores passam por formações iniciais ineficazes que não os capacitam para esse universo. Os autores apontam ainda a vasta proliferação de formações continuadas que buscam amenizar essa lacuna. Fatores psicológicos, motivacionais, estéticos, sociais, ambientais, econômicos e outros são considerados por Galvão Filho (2009) como limitadores para a operacionalização da TA na escola. Fachinetti, Gonçalves, Lourenço (2015) salientam que a formação profissional pode ser um empecilho para a implementação da TA no contexto escolar, e sugerem que a parceria colaborativa entre a professora e a pesquisadora colaborou para a implementação de recursos de TA na escola.

\section{DUA e TA: aproximações e distanciamentos conceituais e práticos}

As considerações tecidas nos itens anteriores nos permitem concluir que o DUA é uma abordagem teórica que, por meio de princípios pré-estabelecidos, busca fornecer subsídios aos professores para que o ensino e o currículo sejam planejados pensando em todos os 
estudantes, enquanto a TA no contexto educacional busca promover soluções individuais para que determinado estudante tenha acesso à aprendizagem, seja por meio da adaptação de um recurso e /ou um serviço.

Percebe-se então que é nesse sentido que se especula uma possível incompatibilidade entre essas duas concepções, afinal, se o DUA parte da premissa que o mesmo currículo deve ser acessível a todos, como conciliá-lo com a TA, que irá promover adaptações específicas a cada estudante?

O início dessa discussão nos reporta aos Estados Unidos, onde o Escritório de Programas de Educação Especial (OSEP) financiou a criação de dois centros de estudos focados em tecnologia. O primeiro denominado Instituto Nacional de Pesquisa em Tecnologia Assistiva (NATRI), da Universidade de Kentucky, e o segundo intitulado Centro Nacional de Acesso ao Currículo Geral (NCAC), em CAST (ROSE; ZABALA; HASSELBRING, 2005).

Rose, Zabala, Hasselbring (2005), em artigo intitulado "Desenho Universal para Aprendizagem e Tecnologia Assistiva: os dois lados da mesma moeda" (tradução nossa), abordam que apesar de se tratar de conceitos distintos, eles não competem entre si, e não precisam ser opostos, afinal, ambas as vertentes estudam maneiras de tornar o ensino acessível a alunos com deficiência.

Isso significa que a utilização dos preceitos do DUA não irá excluir o apoio da TA. Resgatamos aqui que o conceito do DUA nos inspira a pensar no currículo, no ambiente, nos profissionais e nas maneiras de tornar todo o conhecimento acessível a todos os estudantes (ROSE; MEYER, 2014). Ou seja, o foco não está no aluno em si, mas sim no ambiente favorável à sua aprendizagem. Porém, ainda que em determinados momentos as adaptações sob as premissas do DUA possam suprir as necessidades educacionais de todos os estudantes, pode ser que, em outra situação, seja necessária a utilização de uma TA específica para incluir algum aluno em uma atividade.

Um exemplo disso é a utilização de uma bola com guizo para que um estudante com deficiência visual participe de uma atividade na educação física. Na perspectiva do DUA pensamos em como adaptar o ambiente, em planejar a aula, fornecer múltiplos meios de expressão, bem como várias estratégias para que todos os alunos compreendam aquela tarefa (ROSE; MEYER, 2014). Porém, ainda que tenhamos planejados todos os itens meticulosamente, é indispensável que esse aluno participe com uma bola com guizo, afinal, está privado da informação visual, e irá se orientar pela informação auditiva. Nesse momento, a TA surge com uma adaptação para que aquela bola possua sonoridade ao rolar, por exemplo, a bola é envolta em uma sacolinha plástica, permitindo que o aluno consiga se 
orientar pelo barulho. Embora essa adaptação tenha surgido de uma necessidade individual, ela pode ser utilizada por todos os estudantes, portanto, ela não exclui os princípios do DUA, apenas o complementa.

Rose, Zabala e Hasselbring (2005) elaboraram uma figura para exemplificar a relação entre a TA e o DUA (Figura 1). Percebe-se que ambas as vertentes objetivam reduzir barreiras e podem ser utilizadas isoladas ou em conjunto, o que as torna complementares e não excludentes.

Figura 1 - Relação entre Tecnologia Assistiva e Desenho Universal para Aprendizagem

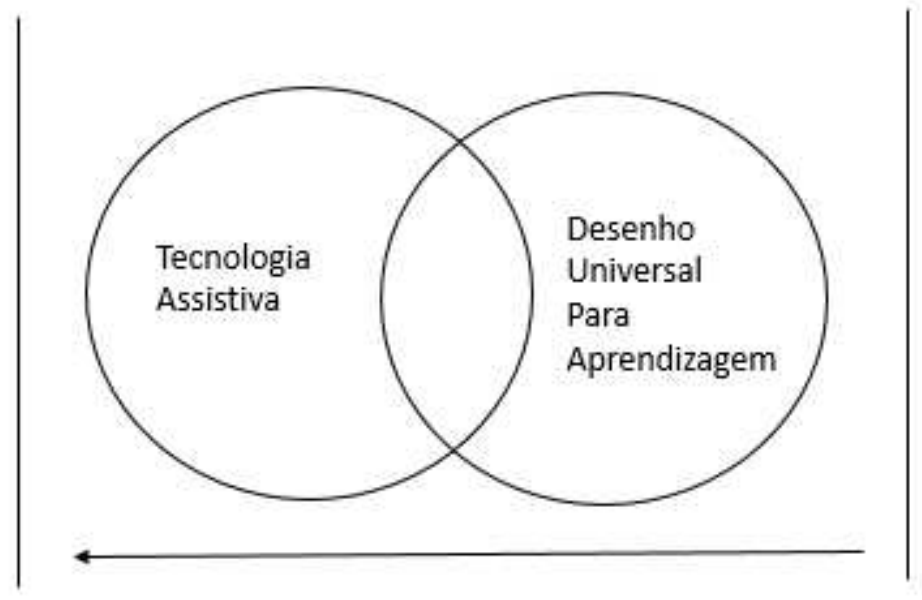

Reduzir barreiras

Fonte: Elaborado pelas autoras - adaptado de Rose, Zabala e Hasselbring (2005)

Nessa mesma perspectiva, Messinger-Willman e Marino (2010) discutem como o DUA pode ser utilizado em conjunto com a TA para aumentar as oportunidades educacionais de alunos com dificuldades de aprendizagem:

A TA e o DUA dependem da tecnologia para melhorar a educação de alunos com deficiências. No entanto, a TA é específica do indivíduo, enquanto o DUA se concentra em uma abordagem holística para o desenvolvimento do currículo. Considere um exemplo em que uma professora de artes da linguagem tem uma aluna da nona série na classe dela. Quando ela vê as dificuldades de aprendizado da aluna do ponto de vista do TA, ela considera como o software de previsão de palavras pode ajudar essa aluna em específico a responder a uma solicitação por escrito. Ao olhar pela lente DUA, ela reconhece que as barreiras de aprendizagem residem em um currículo que força os alunos a escrever respostas manualmente. Ela então altera a avaliação para que a barreira não mais exista para qualquer aluno, permitindo que todos os alunos usem a tecnologia durante suas respostas (MESSINGER-WILMAN; MARINO, 2010, p. 9, tradução nossa). 
Ambas as adaptações realizadas pela professora, nesse caso fictício, seriam eficazes, tanto utilizar o software individualmente, como mudar a forma de avaliação de todos os alunos. Ao concluírem o estudo, as autoras afirmam que a utilização da TA dentro dos preceitos do DUA colaborou para melhorar os resultados acadêmicos e sociais dos alunos com deficiência, mas ressaltam que é necessário que o professor esteja bem orientado e instrumentado para reger essas adaptações (MESSINGER-WILMAN; MARINO, 2010).

Para Alnahdi (2014), o desenvolvimento da TA, sob a ótica do DUA, a torna mais funcional no contexto escolar. O autor aponta o DUA de um lado como uma alternativa para modificar o currículo, o tornando mais acessível a todos, e a TA como complementar a esse currículo, com adaptações mais específicas.

A pesquisa publicada recentemente por Munster, Lieberman e Grenier (2019) objetivou, por meio de um estudo de caso, descrever as diferentes abordagens utilizadas pelos professores para incluir alunos com deficiência em uma escola de educação infantil de Nova York. A análise temática identificou três principais abordagens: (a) instrução normalizada; (b) instrução diferenciada; e (c) instrução baseada nos princípios do DUA. As estratégias mais utilizadas pelos professores foram a instrução diferenciada (aquela adaptada especificamente para um aluno) e as estratégias baseadas no DUA. As autoras concluíram que as duas abordagens são eficazes para acomodar os alunos com deficiência em aulas de educação física.

A instrução diferenciada (ID) abordada pelo estudo explicado no parágrafo anterior, assim como a TA, é uma adaptação específica para cada indivíduo. Entretanto, o próprio Cast (2013) afirma que o DUA e a instrução diferenciada entendem e reconhecem que cada estudante é único e variável, portanto, oferece a flexibilidade de um processo de aprendizagem que considera as necessidades de todos (DUA) e todo (ID) estudante. Nesse sentido, o DUA seria uma teoria que oferece subsídios para pensar no acesso universal à aprendizagem de maneira flexível, ou seja, quando necessárias, as instruções diferenciadas e a tecnologia assistiva são bem-vindas para colaborar com essa aprendizagem.

Mediante esse cenário teórico explanado, no qual as pesquisas na área da TA mostram a tecnologia como uma ferramenta educacional funcional para promover a inclusão, bem como os princípios do DUA como funcionais para um ensino acessível a todos, foi elaborado um quadro (Quadro 1) composto pelas duas vertentes para efeito comparativo. 
Quadro 1 - Comparação entre os preceitos do DUA e da TA

\begin{tabular}{|l|l|l|}
\hline & \multicolumn{1}{|c|}{$\begin{array}{c}\text { Desenho Universal para } \\
\text { Aprendizagem }\end{array}$} & Tecnologia Assistiva como ferramenta educacional \\
\hline Objetivos & $\begin{array}{l}\text { Promover acesso universal ao } \\
\text { currículo e ao conteúdo; oferecer } \\
\text { subsídios teóricos para o professor } \\
\text { planejar um ensino acessível a a } \\
\text { todos. }\end{array}$ & $\begin{array}{l}\text { Promover acesso ao contexto escolar, e atividades } \\
\text { específicas, por meio de adaptações que aumentam } \\
\text { funcionalidade de estudantes com alguma } \\
\text { deficiência e/ou limitação. }\end{array}$ \\
\hline Proposta & $\begin{array}{l}\text { Parte-se do princípio de que o todo } \\
\text { deve se adaptar às necessidades dos } \\
\text { alunos uma vez que cada um } \\
\text { aprende de maneira diferenciada. }\end{array}$ & $\begin{array}{l}\text { Preocupa-se em promover adaptações individuais o estudante com dificuldades possa ter } \\
\text { acesso ao conteúdo, ao currículo e à aprendizagem. }\end{array}$ \\
\hline Adaptação & $\begin{array}{l}\text { Parte de adaptar o geral para o } \\
\text { específico. }\end{array}$ & Parte de adaptar o específico para o geral. \\
\hline Considerações & $\begin{array}{l}\text { A utilização do DUA não exclui a utilização da TA, e vice-versa. Assim como existem } \\
\text { momentos que nenhuma adaptação será necessária, existem momentos nos quais as } \\
\text { adaptações pelos preceitos do DUA serão suficientes. Em outras atividades as adaptações } \\
\text { da TA serão complementares. Assim como a TA pode ser utilizada isoladamente com } \\
\text { sucesso, ou ter a teoria do DUA como sua percussora. }\end{array}$ \\
\hline
\end{tabular}

Fonte: Elaborado pelas autoras

Outra alternativa a se pensar e a ser discutida é quando o uso da TA ganha um caráter universal; essa é uma visão defendida por Almeida (2018):

O recurso de TA é pensado para uma necessidade específica da pessoa com deficiência e em determinada situação, mas muitas vezes, ao ser utilizado também por outras pessoas este recurso pode garantir maior acesso na realização da atividade, ganhando o caráter universal (ALMEIDA, 2018, p. $31)$.

A autora desenvolveu um estudo cujo objetivo foi analisar a implementação de recursos de TA para uma aluna com paralisia cerebral na classe comum e seu uso em caráter universal. Para isso foi realizada uma pesquisa colaborativa que foi subdividida em quatro etapas: entrevista inicial com o professor da classe comum e agente educacional, implementação/intervenção com o recurso de TA por meio do desenho universal na classe comum, avaliação dos recursos de TA implementados na classe comum e entrevista final para encerramento da pesquisa com a professora da classe comum e agente educacional. Após realização de análise de conteúdo a autora indicou que:

[...] os recursos de TA, nos moldes do desenho universal foram utilizados na classe comum e que, na opinião da professora e da agente educacional contribuíram para maior dinâmica na sala de aula e aprendizado para todos os alunos (ALMEIDA, 2018, p. 8).

Em consonância, Cizoto e Francisco (2017) realizaram uma revisão sistemática da literatura com o objetivo de buscar recursos de TA que favoreçam o DUA, "ressaltando onde 
podem ter intersecção concreta e proveitosa para o processo de ensino-aprendizagem" (CIZOTO; FRANCISCO, 2017, p. 7). As autoras pesquisaram na base de dados Scielo combinando os descritores: Tecnologia Assistiva; Desenho universal para a aprendizagem; Escola para todos; Alunos com deficiência, e foram selecionados os artigos que tratavam da TA no contexto educacional e que estivessem entre os anos de 2010-2016. Após analisarem o uso dos recursos de TA no contexto educacional nos artigos selecionados as autoras concluíram que:

Por fim, esta pesquisa destaca que os recursos de TA devem ser pautados nas premissas do DUA. A intersecção da TA e do DUA está na transformação de todo contexto escolar (metodologia, materiais, avaliações entre outros), fomentando e promovendo a sua transformação e forma a otimizar a inclusão de qualidade para todos os alunos, otimizando, assim, o processo de ensinoaprendizagem, diminuindo a evasão escolar e, efetivamente, contribuindo com a consolidação da escola com todos, de maneira concreta. Essa é a forma mais efetiva de viabilizarmos a democracia e a justiça social no seu conceito mais amplo e irreversível (CIZOTO; FRANCISCO, 2017, p. 17).

Percebe-se que, embora haja uma especulação de incompatibilidade entre os conceitos do DUA e da TA, ambos podem ser usados isoladamente e também em conjunto para promover a inclusão educacional, isso porque não existe uma "receita" para promover a inclusão, mas sim é necessário olhar para o aluno, para as necessidades do mesmo, e para o contexto escolar, a fim de viabilizar qual melhor teoria para subsidiar a prática do professor em determinado momento.

\section{Considerações finais}

Subsídios teóricos apontam que tanto o DUA como a TA têm sido utilizados dentro da escola para favorecer o acesso ao currículo tradicional, às atividades propostas e à aprendizagem. Embora sejam abordagens distintas, ambas possuem objetivos consonantes: promover a inclusão educacional. $\mathrm{O}$ fato de pensar em adaptações de maneiras diferentes, sendo o DUA do geral para o específico, e a TA do específico para o geral, não torna as duas vertentes distintas e nem concorrentes, apenas apresentam aos professores e profissionais diferentes maneiras de promover a inclusão na escola.

É importante ressaltar que ambos os conceitos podem ser utilizados juntos para atingir os mesmos objetivos, assim como podem ser utilizados isoladamente e serem eficazes: isso porque cada aluno irá apresentar uma demanda, que será sanada de acordo com suas especificidades. Por exemplo, um aluno que demanda um currículo flexível pode ser 
beneficiado dos princípios do DUA, entretanto, um aluno que precisa de uma adaptação funcional, como um lápis adaptado, terá sua demanda atendida pela TA.

Sugere-se que sejam realizadas pesquisas práticas que utilizem desses dois preceitos para promover a inclusão educacional; dessa maneira será possível verificar como as duas abordagens podem ser utilizadas em consonância para atingir o mesmo objetivo final.

\section{REFERÊNCIAS}

ALMEIDA, R. C. G. O. Desenho Universal para Aprendizagem e Tecnologia Assistiva: implementação de atividades pedagógicas para aluna com paralisia cerebral em classe comum. 2018. 208 f. Dissertação (Mestrado em Educação Especial) - Universidade Federal de São Carlos, São Carlos, 2018. Disponível em:

https://repositorio.ufscar.br/bitstream/handle/ufscar/10449/ALMEIDA_Rita_2018.pdf?sequen $\mathrm{ce}=4$. Acesso em: 02 abr. 2021.

ALNAHDI, G. Assistive technology in special education and the universal design for learning. The Turkish Online Journal Of Educational Technology 18, Arabia Saudita, v. 13, p. 18-23, abr. 2014. Disponível em: https://files.eric.ed.gov/fulltext/EJ1022880.pdf. Acesso em: 11 jul. 2019.

ALVES, A. C. J.; MATSUKURA, T. S. Percepção de alunos com paralisia cerebral sobre o uso de recursos de tecnologia assistiva na escola regular. Rev. Bras. Ed. Esp., Marília, v. 17, n. 2, p. 287-304, 2011.

ALVES, M. M.; RIBEIRO, J.; SIMÕES, F. Universal Design for Learning: contributos de uma escola para todos. Indagatio Didactica, Aveiro, v. 5, n. 4, p. 121-146, 2013. Disponível em: https://proa.ua.pt/index.php/id/article/view/4290/3224. Acesso em: 02 abr. 2021.

BRASIL. Constituição (1988). Constituição da República Federativa do Brasil. Brasília, DF: Senado Federal, 1988.

BRASIL, Declaração de Salamanca, sobre princípios e práticas na área das necessidades educativas especiais. Brasília, DF, 1994. Disponível em:

http://portal.mec.gov.br/seesp/arquivos/pdf/salamanca.pdf. Acesso em: 10 maio 2021.

BRASIL. Lei n. 9.394/96, de 20 de dezembro de 1996. Estabelece as diretrizes e bases da educação nacional. Disponível em: http://www.planalto.gov.br/ccivil_03/leis/19394.htm. Acesso em: 10 maio 2021.

BRASIL. Ministério da Educação. Política Nacional de Educação Especial na Perspectiva da Educação Inclusiva. Brasília, DF: MEC, 2008. Disponível em:

http://portal.mec.gov.br/arquivos/pdf/politicaeducespecial.pdf. Acesso em: 10 maio 2021.

CALHEIROS, D.S.; MENDES, E. G.; LOURENÇO, G. F. Considerações acerca da tecnologia assistiva no cenário educacional brasileiro. Revista de Educação Especial, Santa Maria, v. 31, n. 60, p. 229-244, mar. 2018. Disponível em: https://periodicos.ufsm.br/educacaoespecial. Acesso em: 08 jul. 2019. 
CAST, Center for Applied Special Technology. 2013. Disponível em: http://www.cast.org/udl/. Acesso em: 15 abr. 2018.

CIZOTO, S.; FRANCISCO, J. Tecnologia Assistiva e Desenho Universal para a Aprendizagem: onde se interseccionam? Revista Acadêmica: Ensino de Ciências e Tecnologias IFSP, Cubatão, v. 1, n. 1, p. 1-19, dez. 2017. Disponível em: https://intranet.cbt.ifsp.edu.br/qualif/volume01/ARTIGO01_15_ABNT.pdf. Acesso em: 02 abr. 2021.

FIORINI, M. L. S. Concepção do professor de Educação Física sobre a inclusão do aluno com deficiência. 2011. 143 f. Dissertação (Mestrado em Educação) - Faculdade de Filosofia e Ciências, Universidade Estadual Paulista, Marília, 2011.

GALVÃO FILHO, T. A. Tecnologia Assistiva para uma Escola Inclusiva: Apropriação, Demandas e Perspectivas. 2009. 346 f. Tese (Doutorado em Educação) - Faculdade de Educação, Universidade Federal da Bahia, Salvador, 2009.

HOHMANN, P.; CASSAPIAN, M. R. Adaptações de baixo custo: uma revisão de literatura da utilização por terapeutas ocupacionais brasileiros. Rev. Ter. Ocup. Univ., São Paulo, v. 22, n. 1, p. 10-18, jan./abr. 2011.

LOURENÇO, G. F. Avaliação de um programa de formação sobre recursos de alta tecnologia assistiva e escolarização. 2012. 258 f. Tese (Doutorado em Educação Especial) Centro de Ciências Humanas, Universidade Federal de São Carlos, São Carlos, 2012.

MANZINI, E. J; DELIBERATO, D. Portal de ajudas técnicas para a educação, equipamento e material pedagógico para educação, capacitação e recreação da pessoa com deficiência recursos pedagógicos adaptados II. 1. ed. Brasilia, DF: MEC/ABPEE, 2007.

MASSARO, M.; DELIBERATO, D. Uso de sistemas de comunicação suplementar e alternativa na Educação Infantil: percepção do professor. Revista de Educação Especial, Santa Maria, v. 26, n. 46, p. 331-350, maio 2013. Disponível em: https://periodicos.ufsm.br/index.php/educacaoespecial/article/viewFile/4821/pdf. Acesso em: 23 abr. 2021.

MESSINGER-WILLMAN, J.; MARINO, M. T. Universal Design for Learning and Assistive Technology: Leadership Considerations for Promoting Inclusive Education in Today's Secondary Schools. Nassp Bulletin, v. 94, n. 1, p. 5-16, 2010.

MUNSTER, M. A.V.; LIEBERMAN, L. J.; GRENIER, M. A. (Universal Design for Learning and Differentiated Instruction in Physical Education. Adapted Physical Activity Quarterly, p. 1-21. jun. 2019.

NELSON, L. L. Design and deliver: planning and teaching using universal design for learning. Paul. H. Brookes Publishing Co., 2014. 115 p.

ROCHA, A. N. D. C.; DELIBERATO, D.; LAMÔNICA, D. A. C. Tecnologia assistiva para a criança com paralisia cerebral na escola: identificação das necessidades. Revista Brasileira 
de Educação Especial, Marília, v. 18, n. 1, p.71-92, mar. 2012. Disponível em: https://repositorio.unesp.br/bitstream/handle/11449/117885/S141365382012000100006.pdf?sequence=1. Acesso em: 08 jul. 2019.

ROSE, D. H.; MEYER, A. Teaching every student in the digital age: Universal design for learning. Alexandria: ASCD, 2014.

ROSE, D.; ZABALA, J.; HASSELBRING, T.S. Assistive Technology and Universal Design for Learning: Two Sides of the Same Coin. Handbook Of Special Education Technology Research and Practice, p. 507-518, 2005. Disponível em:

https://www.semanticscholar.org/paper/Assistive-Technology-and-Universal-Design-for\%3A-Two-Rose-Hasselbring/4506ed4fc568cc1640fa48b777a296f730flc1a1. Acesso em: 08 jul. 2019.

SEABRA JUNIOR, M. O. Estratégias de ensino e de recursos pedagógicos para o ensino do aluno com deficiência visual na atividade física adaptada. 2008. Tese (Doutorado em Educação) - Faculdade de Filosofia e Ciências, Universidade Estadual Paulista, Marília, 2008.

TOYODA, C. Y.; LOURENÇO, G. F. Educação Inclusiva: o contexto da terapia ocupacional. In: ALMEIDA, M. A.; MENDES, E. G.; HAYASHI, M. C. P. I. (Org.). Temas em educação especial: múltiplos olhares. Araraquara: Junqueira e Marin, 2008. p. 44-52.

UNESCO. Accountability in education: meeting our commitments. Paris: 2018. Disponível em: https://en.unesco.org/gem-report/report/2017/accountability-education. Acesso em: 19 abr. 2020.

VALENTINE, C. B; GOMES, R. B; BISOL, C. A. Inclusão de estudantes com deficiência intelectual: uma revisão sistemática da literatura. Revista Teias, v. 17, n. 46, jul./set. 2016. Disponível em: https://www.epublicacoes.uerj.br/index.php/revistateias/article/viewFile/25502/18552. Acesso em: 28 maio 2019.

ZERBATO, A. P. Desenho universal para aprendizagem na perspectiva da inclusão escolar: potencialidades e limites de uma formação colaborativa. 2018. 298 f. Tese (Doutorado em educação especial) - Universidade Federal de São Carlos, São Carlos, 2018. 


\section{Como referenciar este artigo}

OLIVEIRA, A. R. P.; GONÇALVES, A. G.; BRACCIALI, L. M. P. Desenho universal para aprendizagem e tecnologia assistiva: complementares ou excludentes? Revista IberoAmericana de Estudos em Educação, Araraquara, v. 16, n. esp. 4, p. 3034-3048, dez. 2021. e-ISSN: 1982-5587. DOI: https://doi.org/10.21723/riaee.v16iesp.4.16066

Submetido em: 05/08/2021

Revisões requeridas em: $30 / 10 / 2021$

Aprovado em: 10/12/2021

Publicado em: 30/12/2021 